\title{
مشكلات مهارة الكلام في تعليم اللغة العربية بمدرسة الثانوية الأهلية الإسلامية تنجونج أوبار حسن نولي المركزية بادانج بولاك جولو المقاطعة بادانج لاوس الشمالية \\ ${ }^{1}$ Yunaldi, ${ }^{2}$ Syahlawani Siregar \\ Pendidikan Bahasa Arab IAIN Padangasidimpuan \\ E-mail: ${ }^{1}$ naldi399@gmail.com, ${ }^{2}$ syahlawanisiregar@gmail.com
}

$$
\begin{aligned}
& \text { تعليم مهارة الكالام هو تعليم المهارة الثانية من تعليم المهارة اللغوية الأجنبية وها المشكالات في تعليم مهارة }
\end{aligned}
$$

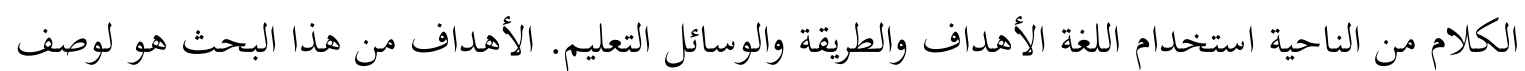

$$
\begin{aligned}
& \text { أنواع المشكلات في تعليم مهارة الكلام باللغة العربية و حل المشكلات تعليم اللغة العربية. المسألة الأساسية } \\
& \text { في هذا البحث هي مشكلات التي يواجه الطلاب. الطريقة المستخدمة في هذا البحث "بحث نوعي. النتائج } \\
& \text { من هذا البحث هي مشكلات تعليم مهارة الكلام باللغة العربية من حيث اللغوية وغير اللغوية. من الناحية } \\
& \text { اللغوية تتكون: مشكلة المفردات، مشكلة المحادثة، مشكلة نظام الصوت، ومشكلة القواعد. وغير اللغوية } \\
& \text { تتكون: مشكلة الرغبة والدافع، مشكلة المرافق والبنية التحتية، مشكلة البيئة. حل لمشكلات تعليم مهارة } \\
& \text { الكلام باللغة العربية اللغة العربية هي إلقاء الخطب العربية في برنامج المحاضرة ، وتوفير حفظ المفردات في كل } \\
& \text { من الفصل وخارجه وتكلم بالعربية بدون استحياء وتشجيع الطلاب تكلم اللغة العربية بطلاقة. } \\
& \text { الكلمة المفتوحة: مشكلات تعليم؛ مهارة الكالام في تعليم اللغة العربية }
\end{aligned}
$$

Abstrak

Pembelajaran maharah al-kalam ialah dari pembelajaran keterampilan yang kedua dari pembelajaran keterampilan Bahasa Asing. Memiliki problematika pada pembelajaran maharah al-kalam di antaranya menggunakan bahasa tujuan, metode dan media pembelajaran. Tujuan dari penelitian ini ialah untuk menjelaskan macam-macam problematika pembelajaran maharah al-kalam dengan Bahasa Arab dan solusinya. Masalah pokok dalam penelitian ini problematika pembelajaran maharah al-kalam yang dihadapi siswa di Madrasah Aliyah Swasta Islamiyah Tanjung Ubar Hasan Nauli kecamatan Padang Bolak Julu kabupaten Padang Lawas Utara. Jenis penelitian ini merupakan penelitian kualitatif. Hasil penelitian menunjukkan bahwa problematika pembelajaran maharah al-kalam dengan Bahasa Arab dari segi linguistic meliputi: Problem Kosakata (Mufradat), Problem percakapan (Muhadatsah), Problem tata bahasa (Qawaid). Sedangkan problematika dari segi non linguistik yaitu meliputi: problem fisiologi, psikologi yaitu meliputi minat, motivasi dan lingkungan. Solusi untuk mengatasi problematika tersebut yaitu: menggunakan metode yang lebih menarik dan menambahkan jam pelajaran bahasa Arab di luar jam kelas. Membuat pidato Bahasa Arab setiap muhadarah, memberikan hafalan mufradat di dalam maupun di luar kelas, mengaplikasikan Bahasa Arab tanpa malu, memotivasi siswa supaya berbicara menggunakan Bahasa Arab.

Keyword: Problematika pembelajaran; Maharat al-kalam Bahasa Arab 
اللغة هي وعاء الثقافة، بمعنى أن اللغة من أهم وسائل الإتصال بين بنى الإنسان، ونتائج حاجته في جماعة انسانية تستلزم منه أن يفهمها، وينتفع بها، ويتكيف معها بكل أنواع العلوم والمعارف. اللغة العربية هي اللغة التي يتعامل بها المسلمون في أمور دينهم، سواء أكانوا يتكلموغا، أم لا. فقراءة القرآن لا تتم إلا بها.' واصطلاحا: عند ابن جني في تعريف اللغة: أصوات يعبر بها كل قوم عن أغراضهم.

واللغة العربية لها أربع مهارات، وهي مهارة الإستماع، مهارة الكلام، مهارة القراءة، ومهارة الكتابة. إن اللغة العربية هي لغة مؤثرة أو مهمة في المدارس الثانوية الإسلامية. من حيث تعليم اللغة العربية، مهارة الكلام هي واحدة من المهارات التي يجب أن يتقن. لأن الغرض الرئيسي من تعلم اللغة العربية هو أن يتمكن الطلاب من التحدث يومياً باللغة العربية وقراءة القرآن في الصلاة بشكل صحيح. مهارة الكلام هي ملكة المتكلم لتعبير ما "يجول في بالها بألفاظ مع تركيب صحيح وبكلام فصيح. المهارة لغة: عرفها ابن منظور بقوله: الحذق في الشيء، والماهر الحاذق بكل عمل. واصطلاحا: شيء يمكن تعلمه أو اكتسابه أو تكوينه لدى المتعلم عن طريق المحاكة والتدريب، وما يتعلمه يختلف باختلاف نوع المادة وطبيعتها وخصائصها والهدف من تعلمه.؛ والمراد بمهارة الكلام هنا وهو لغة منطوقة للتعبير عن أفكار ذهنية. واللغة في الأساس هي الكلام. يقصد ' ابراهيم محمد عطا، طرق تدريس اللغة العربية والتربية الدينية (جامعة القاهرة: مكتبة النهضة المصرية، إعاهـ- 997 (1))،

$$
\begin{aligned}
& \text { ص. }
\end{aligned}
$$

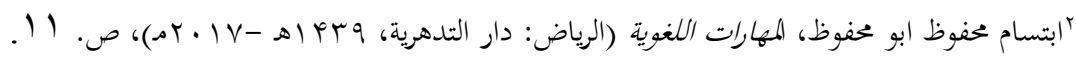

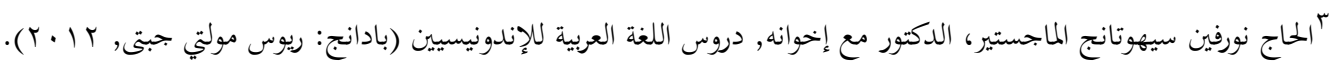

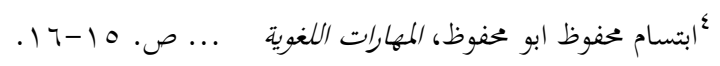




\section{Vol. 9. No. 2 Desember 2021}

بمهارة الكلام أو غالبا ما يطلق عليه بتعبير. هو مهارة لنقل مهارة شفويا إلى الآخرين. فإن كلا من التركيز على مختلف الإمكانات الشفوية. أن تتحقق التعبير شفويا أو كتابيا.

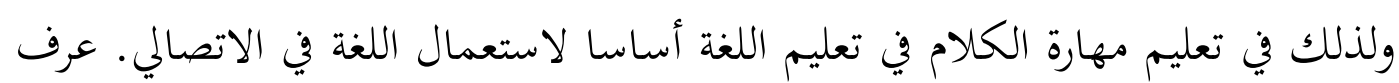
أن التعليم هو كل ما يمكن أن يكتسب المعلومات والمعرفة في التفاعل الذي يحدث بين المعلمين والطلاب. تعليم اللغة العربية هو عملية تنظيم تفاعل المتعلمين مع المعلمين وموارد التعلم في بيئة تعليمية لتحقيق أهداف التعلم بنشاط وكفاءة حتى تحصل عملية التعلم باللغة العربية على نتائج جيدة.

زاد محمد مزمل في كتابه أن التعليم هو عملية توفير الشروط المادية والنفسية التي تساعد

التلميذ على التفاعل النشط مع عناصر البيئة في موقف محدد، يقوم بهذه العملية معلم مدرسا مستخدما أبسط الطرق الممكنة. v وهذه المهارة مهمة. وأهمية مهارة الكلام يعنى هو من المهارات الأساسية، التي يسعى الطالب إلى إتقاها في اللغات الأجنبية، ولقد اشتددت الحاجة إل هذه المهارة في الفترة الأخيرة، عندما زادت أهمية الاتصال الشفهي بين الناس. ومن الضرورة بمكان عند تعليم اللغة العربية الاهتمتم بالجانب الشفهي، هذا الاتحاه الذي نرجو يسلكه معلم اللغة العببية، وأن يجعل همه الأول تمكين الطلاب من التكلم بالعبية، لأن العربية اتصال، يفهمها ملايين الناس في العالم.^ وأهداف تعليم مهارة الكلام هو يتضمن الغرض من تعليم مهارة الكلام عدة أشياء، منها: أ) سهولة الكلام؛ يحتاج الطلاب إلى تطوير إمكاناقم في مهارة الكلام بثقة من خلال تمارين متتعة إما مع الأصدقاء أو ايّ مكان ما، ب) الوضوح؛ لأفما ممارسة مهارة الكلام بشكل

$$
\text { 0عبد الرحمن ابراهيم الفوزان، إضاءات لمعلمي اللغة العربية...ص. } 9 .
$$

†البيرت ابيندي، Konsep Pembelajaran Daring Berbasis Pendekatan Ilmiah (جاوة الوسطى: CV Sarnu 
صحيح، فإنها تأحذ بحموعة متنوعة من التمارين المستمرة والمتنوعة، ج) المسؤولة ؛ في هذه التدريب، سيتم منع شخص من الكلام بشكل غير مسؤول، وإنشاء جلسة استماع حرجة؛ في هذه المسألة، يحتاج الطلاب إلى تعليم تقييم الكلمات التي تم نطقها، وينبغي أن يكون القصد عند القول والغرض، د) الإنشاء؛ إن كل ما يتطلبه الأمر هو الالتزام بخلق هذه العادة الناطقة باللغة العربية، فإن هذا الالتزام يتطور اتفاق الآخرين باللغة العربية بشكل كبير. تسمى بيئة اللغة الحقيقية. استعمال الطريقة المناسبة في تعليم المهارات مهم جدا، والطريقة وهي خطة عالمية تتعلق بالعرض المنظم أو المنهجي للمواد اللغوية على مدخل خاص. 'ا في هذا البحث، سيكون من الطرق التي تعتبر مشهورة وهي طرق أساسية، منها: أ) الطريقة المباشرة، وتركيز هذه الطريقة أكبر على اللغة المنطوقة وعدد من تقنياتا. طريقة المباشرة لما الإجابيات والسلبيات، اجابياها:أ) الطلاب في مهارة الاستماع ومهارة الكلام، ب) اتقان الطلاب بلفظ صحيح، ج) يتمتع الطلاب بثقة للتواصل اللغة العربية، د) القواعد يدريس الطلاب هو قواعد. وعيوب هذه الطريقة منها: يطبق في بحموعة صغيرة، ثم الطلاب ضعفاء في مهارة القراءة، ويحتاج إلى معلم ماهر وبطلاقة. ب) طريقة السمعية والبصرية ومن أهم أسس هذه الطريقة استعمال الوسائل السمعية والبصرية بصورة، مكثقة، واستخدام أساليب متنوعة لتعليم اللغة، مثل المحاكة والترديد والاستظهار، والتركيز على طريقة القياس، التقليل من الشرح، والتحليل النحو. إيجابياها: بتركيزها على الكلام تؤكد على الجانب الاتصال في اللغة، ثم تهتم بثقافة اللغة، والتفكير باللغة، واستخدام الوسائل، والاستخدام بالتدريبات، والتدرج في تقديم اللغة، وضبط المفردات والتراكيب المقدمة للمتعلم، وسلبيات هذه الطريقة منها: التكرار أمر جيد ولكن هناك أساليب أسرع يجب

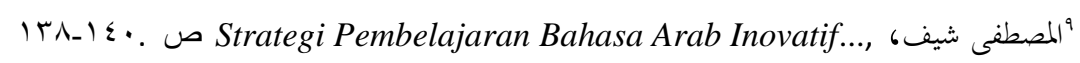

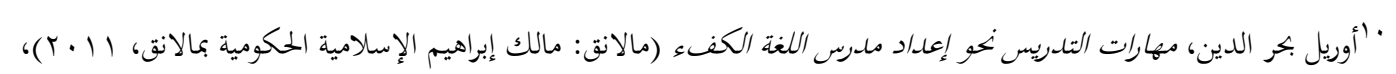


عدم بتحاهلها، والفصل بين المهارات اللغوية لا يلي حاجات الدارسين كافة، ثم عدم مراعاة الفروق الفردية (الجميع يسمعون. والجميع يرددون)، والاهتمام بالاستماع والكلام على حساب مهارتي القراءة والكتابة.ج) طريقة القراءة؛ خصائص استخدام طريقة القراءة التالية: تفتح هذه الطريقة من خلال إعطاء الطلاب لحظة ممارسة على مهارة الكلام والاستماع على عدة البسيطة ونطق الكلمات والجمل حتى يتمكن الطلاب من تأليف الجمل، وتقسم القراءة إلى قسمين، أي القراءة المكثفة والقراءة المستقلة، ولكل منهما غرض مختلف، ثم المقصود من القراءة الجهرية هي

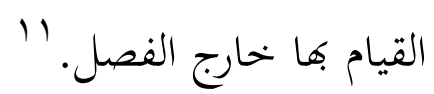

بناء على بيان السابق، يخلص أن طرق تعليم مهارة الكلام باللغة العربية الذي يبحث في هذا البحث لها خمسة طرق، منها: طريقة القواعد والترجمة، طريقة المباشرة، طريقة السمعية والبصرية، طريقة القراءة.

ومن صعوبات التي يواجهها الطلاب في عملية التعليم الناجمة عن خصائص اللغة العربية

بلغة أجنبية منها؛ أ) نظام الصوتي، يتميز النظام الصوتي في اللغة العربية بخصائص مختلفة ويختلف في طريقة نطقه، ولكل منها خصائصه الخاصة. ب) المفردات؛ عامل مفيد لمتعلمي اللغة العربية ومعلمي اللغة العربية في إندونيسيا هو جانب المفردات لأن الكلمات العربية دخلت اللغة الإندونيسية أو الإقليمية. فإن نقل الكلمات من اللغة الأجنبية إلى لغات الطلاب أن يسبب مشكلات. ج) النحو والصرف؛ وهو من ناحية الإعراب وتغيير صغة الكلمات في الجمل. بناء على نتائج المقابلات والملاحظات الأولية التي أجريت في اليوم أو التاريخ الخميس ب

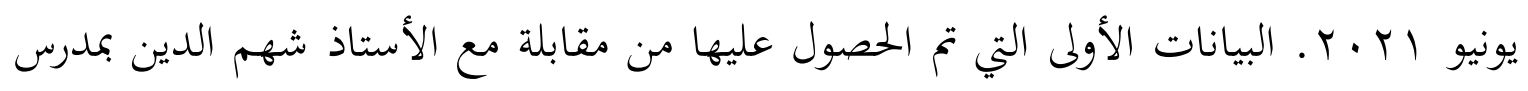
في بحال العربية في مدرسة الثانوية الأهلية الإسلامية تنجونج أوبر حسن نولي قال إنه إذا نتكلم 
عن المشاكل العربية من التحديات التي تواجهها الآن، المشكلة التي يواجهها الطلاب عند الكلام باللغة العربية هي عدم إتقان الموفرات والتشجيع والثقة والتدريب. rا والسبب هو عدم الممارسة مع اللغة العربية. عندما يتعلق الأمر بأساليب التعلم العربية،

فإن الطريقة التي أستخدمها هي طريقة القواعد والترجمة. بالنسبة حصة لمادة اللغة العربية حصتين فقط في أسبوع. المدرسة الثانوية الأهلية الإسلامية تنجونج أوبار حسن نولي المقاطعة بادانج بولاك جولو المركزية بادانج لاوس الشمالية. العدد الإجمالي لمعلمي اللغة العربية هناك معلمان، أحدها في مدرسة المتوسطة و مدرسة الثانوية الأهلية الإسلامية تنجونج أوبار حسن نولي. منهاج البحث

منهج هذا البحث هو البحث الميداني. "الميداني" (البحث الميداني) في الإثنوغرافيا يعني أن الباحثة يجمع البيانات في البيئة التي يوجد فيها المشترك وحيث دراسة الأنماط الثقافية. با" الغرض من هذا البحث هو بناء نظرية وهي أفكار جديدة من أجل اشتقاق المعنى من البيانات ومناقشة نتائج البحث.

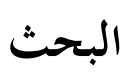

النتائج من هذا البحث هي وجد المشكلان في تعليم اللغة العربية عاما. ومن مشكالات

اللغوية، وهي علم اللغة التي تتم علمية. في تطورها خضع اللغوية لعملية اشتقاق متنوعة جدا وواسعة النطق. اللغوية التاريخية هي لغوية تحقق في تغيير الطويلة وقصيرة الأجل في نظام الصوت والقواعد والمفردات.؛ ا بناء على البحث التي تم إجراؤها، وجدت بعض المشكاتلات تعليم مهارة باشهم الدين ، معلم اللغة العربية في مدرسة الثانوية الأهلية الإسلامية تنجونج أوبار حسن نولي في التاريخ 05 ,

يوني.2021.

, Metode Penelitian Pendidikan Pendekatan Kualitatif, Kuantitatif dan Penelitian نأمدار

. Media, 2016), : Citapustaka بندونج Pengembangan (

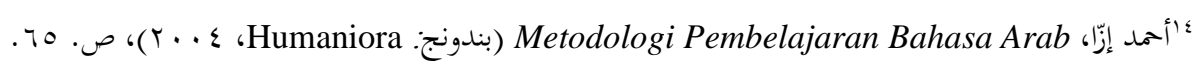




\section{Vol. 9. No. 2 Desember 2021}

الكلام باستخدام اللغة العربية لا يحدث فقط في الطلاب ولكن من الجانب الآخر أيضا يخالفة مثل مشكلات المعلم، ومشكلات النظام الصوت، والمشكلات النحوية والمفردات وبصورة عامة، توصف المشكلة التالي: أ) نظام الصوت، وهذا القسم يصبح الأساس في إتقان مهارة الاستماع ومهارة الكلام. 10 هو مشكلات الصوت، منها: بعض أصغر صوت للغة تميز المعنى. الإندونيسية ليس لها ما يعادلها باللغة العربية.

بناء على نتائج تسجيل طلاب مدرسة الثانية الأهلية الإسلامية تنجونج أوبار حسن نولي، وجدت الباحثة أن الحروف الساكنة التي يصعب نطقها، هي الطلاب يجدون صعوبة في نطق بعض الحروف الحجاءية التي تبدو متشابهة تقريبا، مثل السين والثاء، الصاء والزاي. وبناءا على المقابلة التالي: عدم ممارسة النطق بالصوت الساكن يتفق مع ما يدرسه المعلم وعدم معرفة

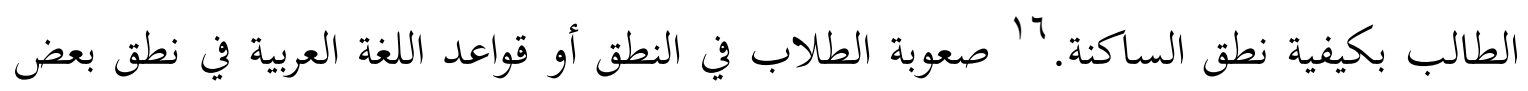
الأصوات الساكنة بعدم ممارسة النطق الساكن وفقا لما يدرسه المعلم. الأول: النحوية؛ وفي تعليم اللغة العربية، قواعد اللغة هي العنصر الأول الذي يجب أن يتقنه تعليم مهارة الكلام اللغة العربية. من قبل الخبراء العرب، يتم وضع العلم النحو هو مركز لجميع الخصص، حيث ينطبق علم النحو والده وينطبق علم صرف أمه. في عملية التقديم، هذه الطريقة مفيدة جدا للطلاب في معرفة موقف الإعراب. وفقا للأخت ريسكا سافيتري: "أشعر بالفراحة وفقا للمادة العربية. لكنني أجد صعوبة من القواعد. لأنني لا أفهم القواعد". نتائج المقابلة مع الأخت اسرا ديوي: صعوبة تعليم مهارة الكلام باللغة العربية هي أن الأخت لا تفهم عن النحو. لأنه في تعليم اللغة العربية يجب أن يفهم أسلوب نحو صرف ليكون

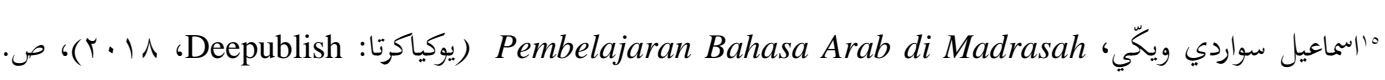


أسهل في تنفيذ تعليم اللغة العربية.^ا' وعلى النقيض مما قالته الأخت ليسفي دوارين: "هل دروس اللغة العربية سهلة؟ فأجابت: "دروس اللغة العربية صعبة". 19

ومن النتائج المقابلات السابقة، أن الطلاب تقريبا مثل المواد العربية. ويمكن الاستفادة من

اهتمامهم في إعداد الأدوات التي تدعم التعليم. كيفية تعليم باستخدام "طريقة النحو الترجمة"، اجعلهم يشاركون بنشاط التعليم. بحيث لا يشعرون المشبعة أن تولي اهتماما للدروس التي وصفها المعلم للاهتمام. فإن عامل الطلاب في مشكلة القواعد داعمة جدا بمدرسة الثانوية الأهلية الإسلامية تنجونج أوبار حسن نولي.

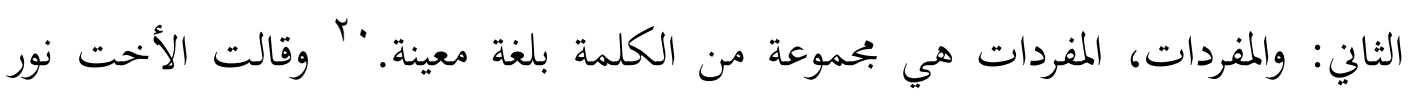

هواني: "لا أتكلم باللغة العربية، ما أستخدمها عادة هي اللغة الدائرية. وعدم إتقان المفردات العربية التي حفظتها". "بيختلف الأخ أنخس مارجوكي:"نعم، أتكلم باللغة العربية ولكن غير طلاقة، لأن الانغماس في المفردات لا يزال قليلا، نادرا ما تستخدم في الأنشطة اليومية، تطبق

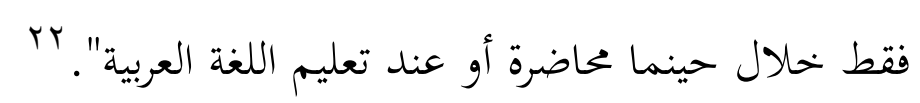

المشكلات التي يواجهها الطلاب عند تكلم اللغة العربية في مدرسة الثانوية الأهليه

تنجونج أوبار حسن نولي منها: عدم إتقان المفردات والافتقار إلى المرافق والبنية التحتية مثل

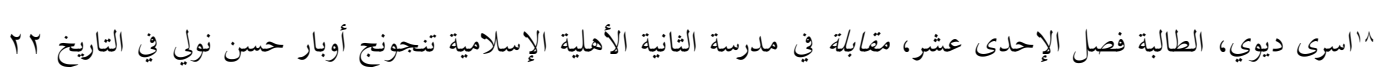

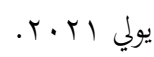
'باليسفي دورني، الطالبة فصل الإحدى عشر، مقابلة في مدرسة الثانية الأهلية الإسلامية تنجونج أوبار حسن نولي في التاريخ YY

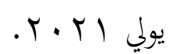

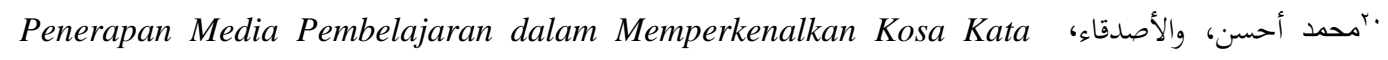
Bahasa Arab

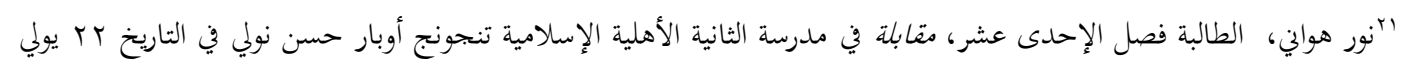
IV Yr Y Y Yخاس مرزوكي ، الطالب فصل الإحدى عشر، مقابلة في مدرسة الثانية الأهلية الإسلامية تنجونج أوبار حسن نولي في التاريخ 
غختبر اللغة، فضلا عن تعليم وسائل الإعلام التي بتعل الطلاب أكثر حماسا في تعليم اللغة العربية والتحفيز من المعلم والولي الأمور والبيئة على حد سواء.

وأما مشكلات غير اللغوية منها ؛ مشكلات تعليم مهارة الكلام باللغة العربية في التحفيز واهتمام الطلاب والمرافق والوسائل المسبقة ، وفي علم النفس العام ، يمكن أن يكون معروفا هو الدافع الذي يستند إلى ظهور السلوك ، يقصد هذا الدافع بقوة دافعة للقيام بشيء معين. بَّ أن يقال الاهتمام بسبب، أي القوة الدافعة التي بتحبر المرء على تقسيم الانتباه بشأن الحالة أو الأنشطة الخاصة وليس الآخرين. إذا كان طلاب المدرسة الثانوية من مدرسة المتوسطة قد درسوا اللغة العربية، فإها تميل إلى أن تكون سهلة ومتحمسة ومهتمة بتعليم مهارة الكلام باللغة العربية في مدرسة الثانوية. والعكس، إذا كان طالب المدرسة الثانوية يتعلم اللغة العربية هذه المرحلة لأنه خريج مدرسة ثانوية حكومية، فيجب أن يواجه صعوبات في تعليم مهارة الكلام باللغة العربية لأهما تجربته الأولى والدوافع واهتماماته تختلف عن الآخرين من المجموعة الأولى. بناء على مقابلة الباحثة مع المخبر:الطلاب الذين ليس لديهم دافع قوي في تعلم مهارة

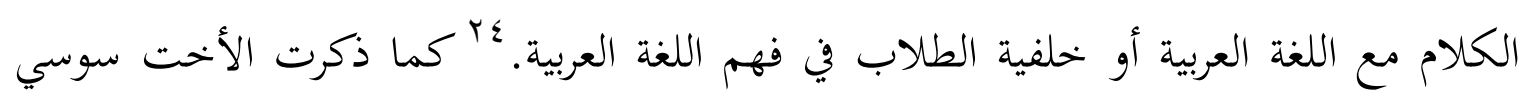

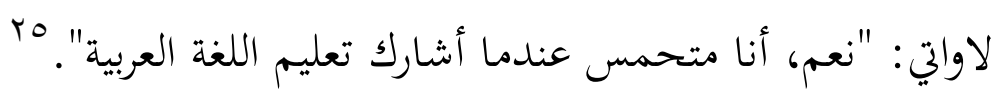
وقالت مخبر آخر: "أنا أقل اهتماما باللغة العربية وأقل حماسا للمشاركة في تعليم اللغة

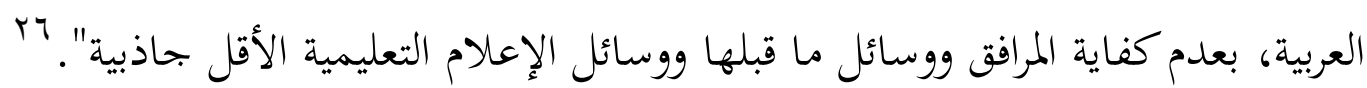

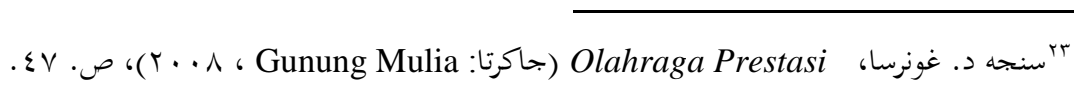

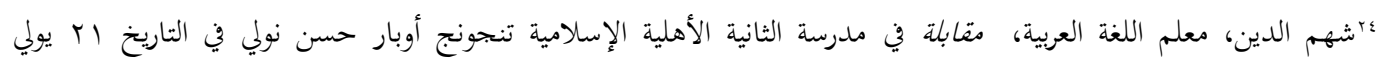
•r بوسي لواتي ، الطالبة فصل الإحدى عشر، مقابلة في مدرسة الثانية الأهلية الإسلامية تنجونج أوبار حسن نولي في التاريخ Tr

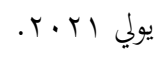

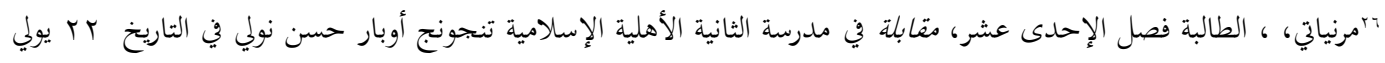


"إن الوسائل والمرافق المسبقة التي يستخدمها المعلم في تعليم مهارة الكلام باللغة العربية

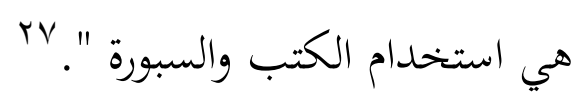
بناء على نتائج المقابلة السابقة، هناك بعض الطلاب الأقل حماسا للمشاركة في تعليم مهارة الكلام باللغة العربية بعدم كفاية المرافق والمرافق المسبقة ووسائل الإعلام التعليمية الأقل جاذبية. فيها حاجة التعليم الوسائل الإعلام والوسائل المسبقة في عملية التعليم. من أجل زيادة اهتمام الطلاب وتحفيزهم عند التكلم باللغة العربية. ثم المشكلات البيئة؛ أحد العوامل التي تؤثر بشكل كبير على بنحاح عملية التعليم وتحديده هي البيئة، ليس أقلها البيئة اللغوية. إن وجود بيئة ناطقة باللغة العربية تصبح مهما جدا لأها موجود دائما، تعطي فارقا بسيطا في نفس السياق تعليم مهارة الكلام باللغة العربية. هذه أهمية البيئة التعليمية. توفر بيئة المدرسة فرصة كبيرة للطلاب ليتمكنوا اللغة العربية ولكن المدرسة لا لوائح تشجع الطلاب أن يجعل اللغة العربية لغة تتم تطبيقها في الأنشطة اليومية. مقابلة صاحبة البحث مع المخبر أن:

المشكلات التي أواجهها عند الكلام باللغة العربية هي عدم الاهتمام والتحفيز والبيئة وعدم إتقان المفردات. ^^ يعرف أن أهمية خلق بيئة لدعم عملية تعلم اللغة العربية، وخاصة في اللغة العربية. لأن الشخص الذي هو الدافع الكبير سيكون عنيد للتعلم ولا يلين، وقراءة الكتب بجد لتحسين إبحازاته للتغلب على المشكلة.

لانور هواني ، الطالبة فصل الإحدى عشر، مقابلة في مدرسة الثانية الأهلية الإسلامية تنجونج أوبار حسن نولي في التاريخ ب يولي tr. rI ^זتيا ريف ، الطالبة فصل الإحدى عشر، مقابلة في مدرسة الثانية الأهلية الإسلامية تنجونج أوبار حسن نولي في التاريخ ب يولي 


\section{Vol. 9. No. 2 Desember 2021}

وقالت الأخت إيسراء ديوي: "أنني لا أتكلم باللغة العربية بعد لأنني لا أفهم اللغة العربية جيدا ولا أطبق اللغة العربية داخل أو خارج الفصل". وب والمدرسة التي تتطلب من الطلاب باللغة العربية كل يوم ستساعد طلابهم على إتقان المفردات.

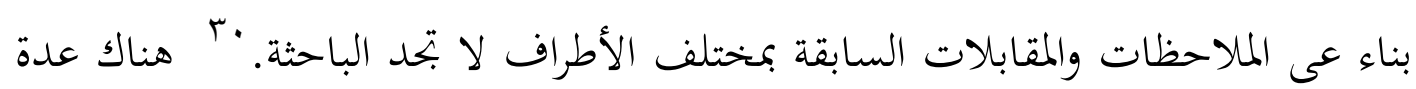

عوامل تشمل على الرغم من أن المدرسة الدينية هي نموذج للإسلام، ولكنها لم تطلب من الطلاب استخدام اللغة العربية بلغة رسمية. إذا كان أي شخص يتكلم باللغة العربية بوسيلة تواصل بين الطلاب والطلاب، والطلاب مع المعلم هو بحرد استعداد.

الخحلاصة

الخلاصة من هذا البحث هي وجد مشكلان في تعليم مهارة الكلام وهو مشكلة اللغوية ومشكلة غير اللغوية. ومن مشكلة اللغوية هي تتكون من مشكلة نظام الصوت والقواعد والمفردات للغة نظام الصوت، وأما مشكلة غير اللغوية تتكون من مشكلات الاهتمام والتحفيز، ومشكلات المرافق والبنية التحتية والمشكلات البيئة.

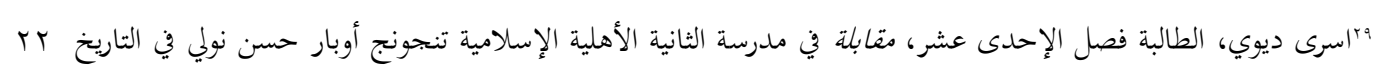




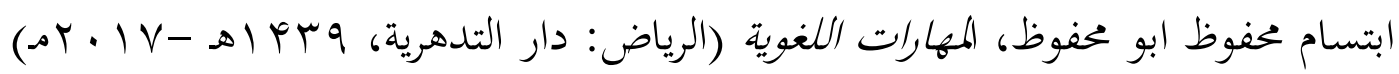
ابراهيم محمد عطا، طرق تلدريس اللغة العربية والتربية الدينية (جامعة القاهرة: مكتبة

$$
\text { النهضة المصرية، } 19 \text { (1 (هـ- } 997 \text { (مـ). }
$$

أحمد إزّا، Metodologi Pembelajaran Bahasa Arab (بندونج: Humaniora، ع . . ؟ ) , Metode Penelitian Pendidikan Pendekatan Kualitatif, Kuantitatif dan أحمد نذار Media, 2016) : Citapustaka بندونجPenelitian Pengembangan ( أحمدي، معلم اللغة العربية، مقابلة في مدرسة الثانية الأهلية الإسلامية تنجونج أوبار حسن نولي

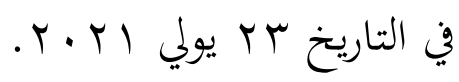

اسرى ديوي، الطالبة فصل الإحدى عشر، مقابلة في مدرسة الثانية الأهلية الإسلامية تنجونج

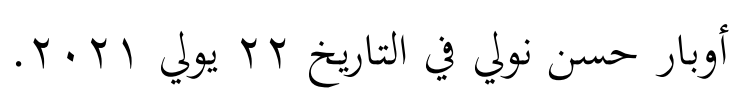

اسرى ديوي، الطالبة فصل الإحدى عشر، مقابلة في مدرسة الثانية الأهلية الإسلامية تنجونج

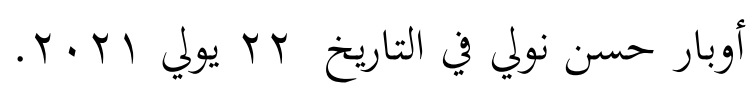

اسماعيل سواردي ويكّي، Pembelajaran Bahasa Arab di Madrasah $(\uparrow \cdot) \wedge$ เDeepublish أبحاس مرزوكي ، الطالب فصل الإحدى عشر، مقابلة في مدرسة الثانية الأهلية الإسلامية

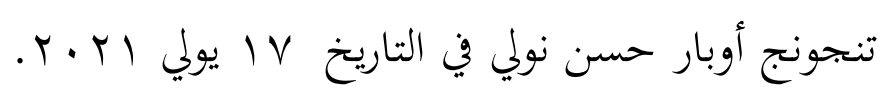
أوريل بحر الدين، مهارات التدريس نحو إعداد مدرس اللغة الكفء (مالانق: مالك إبراهيم الإسلامية الحكومية بمالانق، 11 (ب) بصري مصطفى، عبد الحميد، ... 
البيرت ابيندي، Konsep Pembelajaran Daring Berbasis Pendekatan Ilmiah (جاوة الوسطى:

$$
(Y \cdot T \cdot \text { GCV Sarnu Untung }
$$

تيا ريف ، الطالبة فصل الإحدى عشر، مقابلة في مدرسة الثانية الأهلية الإسلامية تنجونج أوبار

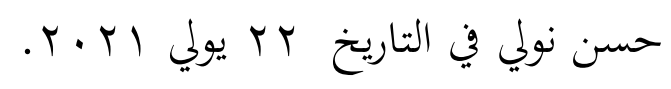

الحاج نورفين سيهوتانج الماجستير، الدكتور مع إخوانه, دروس اللغة العبية للإندونيسيين

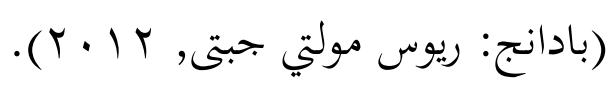

رسكا سافتري، الطالبة فصل الإحدى عشر، مقابلة في مدرسة الثانية الأهلية الإسلامية تنجونج

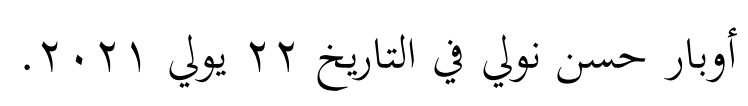

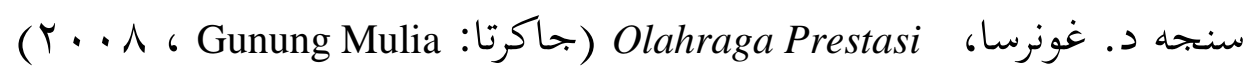
سوسي لواتي ، الطالبة فصل الإحدى عشر، مقابلة في مدرسة الثانية الأهلية الإسلامية تنجونج

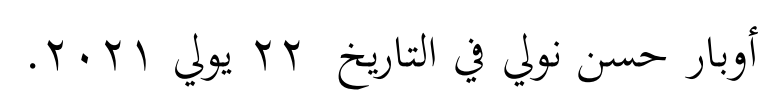

شهم الدين ، معلم اللغة العربية في مدرسة الثانوية الأهلية الإسلامية تنجونج أوبار حسن نولي

$$
\text { في التاريخ } 05 \text {, يوني.2021. }
$$

شهم الدين، معلم اللغة العربية، مقابلة في مدرسة الثانية الأهلية الإسلامية تنجونج أوبار حسن

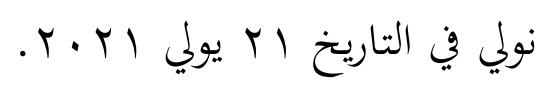

عبد الرحمن ابراهيم الفوزان، إضاءات لمعلمي اللغة العربية

عبد الرحمن ابراهيم الفوزان، إضاءات لمعلمي اللغة العربية لغير الناطقين بها، (مالك إبراهيم

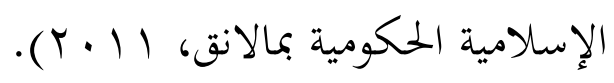

ليسفي دورني، الطالبة فصل الإحدى عشر، مقابلة في مدرسة الثانية الأهلية

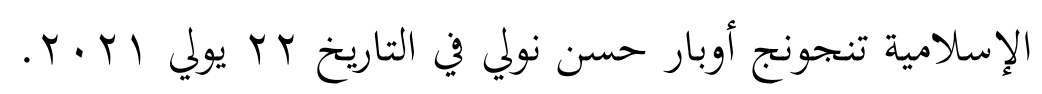


Penerapan Media Pembelajaran dalam Memperkenalkan Kosa محد أحسن، والأصدقاء، Nusantara باريباري: الجامعة الإسلامية الحكومية باري باري Kata Bahasa Arab

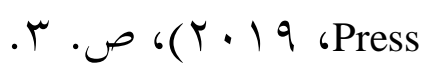

حممد مزمل البشير ومحمد مالك محمد سعيد، ملدخل إلى المناهج وكرق التدريس (الرياض:

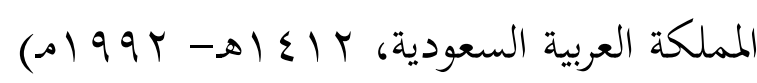

مرنياتي، ، الطالبة فصل الإحدى عشر، مقابلة في مدرسة الثانية الأهلية الإسلامية تنجونج أوبار حسن نولي في التاريخ ب ب يولي اب • ب.

Strategi Pembelajaran Bahasa Arab Inovatif. المصطفى شيف، نور هواني ، الطالبة فصل الإحدى عشر، مقابلة في مدرسة الثانية الأهلية الإسلامية

$$
\text { تنجونج أوبار حسن نولي في التاريخ بr يولي اب ·r. }
$$

نور هواني، الطالبة فصل الإحدى عشر، مقابلة في مدرسة الثانية الأهلية الإسالامية تنجونج

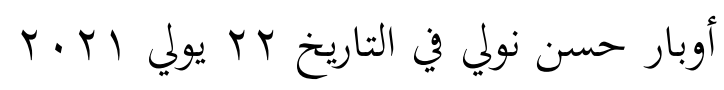

